\title{
Comportement du sol au labour : évolution de l'état structural au cours du labour
}

\author{
I Coulomb 1, J Caneill 2*, H Manichon ${ }^{3}$ \\ avec la collaboration technique de M Tremblay \\ 1 INRA Agronomie, F78850 Thiverval-Grignon; \\ 2 INA-PG, 16, rue Claude-Bernard, F75231 Paris cedex 05; \\ ${ }^{3}$ CIRAD, BP 5035, F34032 Montpellier, France
}

(Reçu le 7 octobre 1992; accepté le 7 avril 1993)

\begin{abstract}
Résumé — Le rôle de l'état initial du sol vis-à-vis des processus de fragmentation par la charrue à socs est étudié expérimentalement en sol limono-argileux à comportement fragile. À cet effet, on compare les évolutions de trois états structuraux initiaux différents au cours du labour. Après discussion de la compatibilité des volumes de sol caractérisés avant et après labour, qui doivent être représentatifs du volume de sol réellement travaillé par la charrue, l'évolution de l'état structural du sol est quantifiée grâce à un bilan surfacique et numérique des différentes catégories de mottes. Les résultats montrent que les transformations d'état au cours du labour sont dépendantes de l'état structural du sol avant labour. Quel que soit le traitement, le volume des mottes compactées ne varie pas. L'augmentation du volume apparent du sol au cours du labour est liée à une augmentation du volume cumulé des mottes poreuses et de la terre fine, accompagnée, lorsque l'état initial est continu et compacté, de l'apparition de vides supracentimétriques. Lorsque l'état est continu et tassé, le labour modifie aussi la taille et l'état de fissuration des mottes. L'ampleur des variations concernées est fonction de la résistance de la couche labourée.
\end{abstract}

labour / état structural / motte / profil cultural

Summary - Behaviour of soil during ploughing: soil structure evolution during ploughing. The function of soil structure before ploughing with regard to mechanical processes during ploughing has been studied. The experiment was carried out in a clay loam soil with friable behaviour. The evolution of 3 different structures before ploughing was compared. The compatibility of soil volumes characterized before and after ploughing was analyzed. Soil structure was characterized by measurement of the area and the greater length of different types of clods (compacted, compacted with cracks, porous), the area of fine earth and macroscopic voids (diameter $>1 \mathrm{~cm}$ ) on a map of the ploughed layer profile. Evolution was assessed via balance of each area and balance of clod number with a given length. Results showed that decrease in ploughed layer density during ploughing is linked to increase of porous clods and fine earth cumulative volume. It is accompanied by the appearance of macroscopic voids, when the soil structure before ploughing is compacted and continuous. In this case, clod size and cracks are modified by ploughing. Clod size reduction is greater when the ploughed layer is less resistant. When the layer is more resistant, fragmentation is not complete. Then appearance of cracks reveals the result of the stress-strain relationship in the clod.

ploughing / soil structure / clod / soil profile

\footnotetext{
* Correspondance et tirés à part
} 


\section{INTRODUCTION}

Le labour est une opération couramment pratiquée en Europe, car il permet l'enfouissement des matières organiques et d'engrais, la destruction des plantes adventices, l'incorporation des reliquats de pesticides et, enfin, il a un effet sur l'état physique du sol et sur l'amélioration de la circulation de l'eau en régime saturé. Nous avons établi dans un article précédent (Coulomb et al, 1993) l'effet déterminant de l'état structural du sol avant labour sur l'état structural du sol après labour sur une parcelle de texture limonoargileuse labourée à une humidité moyenne d'environ $0,20(\mathrm{~g} / \mathrm{g})$. Nous avons montré, dans ces conditions expérimentales, que la vitesse d'avancement du tracteur ne pouvait contribuer à expliquer la variabilité des états après labour. Celle-ci se structure principalement selon les traitements différenciés par leur état structural du sol avant labour. Les différences observées entre les traitements après le labour peuvent résulter de la conservation de la différence d'état initial pour un même travail de fragmentation du sol par la charrue, ou bien d'un travail de fragmentation qui varie selon l'état initial. L'objectif de cet article est donc d'identifier le rôle joué par l'état initial vis-à-vis des processus de fragmentation dans les conditions précédentes. II s'agit de dégager les critères de la structure réellement modifiés par le labour et d'en quantifier l'évolution. Les résultats de l'article cité montrent que le classement avant labour de situations expérimentales selon les volumes occupés par les mottes compactées correspond au classement des situations après labour. La distribution des calibres de ces mottes après labour varie selon la résistance mécanique initiale de la couche labourée et l'état de fissuration des mottes.

Nous pouvons donc formuler les hypothèses suivantes :

- le volume des mottes compactées se conserve au cours du labour ; cette hypothèse a déjà été faite par Manichon (1988) pour analyser l'état structural selon les systèmes de culture ;

- l'augmentation de volume apparent de la couche de sol travaillé au cours du labour ne peut être liée qu'à une augmentation du volume : de la terre fine, des mottes à état interne plus poreux, des vides macroscopiques $(>1 \mathrm{~cm})$;

- la variation du calibre des mottes compactées dépend de l'état structural initial.
Nous avons entrepris de tester ces hypothèses en quantifiant les variations d'état du sol sous l'action du labour sur des situations expérimentales différenciées par leur état avant labour. Nous discuterons des processus de fragmentation pouvant expliquer ces variations.

\section{ANALYSE DE L'ÉVOLUTION : MATÉRIEL ET MÉTHODES}

\section{Conditions expérimentales}

L'expérimentation a été menée en région parisienne sur une parcelle $(85,0 \mathrm{~m} \times 16,2 \mathrm{~m})$ du Centre expérimental de Grignon (78) sur un luvisol orthique avec une couche labourée de texture limono-argileuse $(A=$ $25,9 \% ; L=58,1 \% ; S=16,0 \% ; M O=2,1 \%$ ).

Trois traitements correspondant à trois états différenciés avant labour (NT, T1, T2) ont été créés. T1 et T2 ont été obtenus par des compactages à deux dates différentes après la récolte du précédent et avant labour ( 1 mois avant labour pour T1, 1 semaine avant labour pour T2) alors que NT est exempt de compactage après la dernière récolte et résulte de l'histoire culturale de la parcelle. Les compactages, réalisés avec le même tracteur, ont été suivis d'une opération de nivellement par une herse réglée superficiellement.

Le labour a eu lieu à une humidité moyenne de la couche labourée comprise entre 0,20 et $0,21(\mathrm{~g} / \mathrm{g})$, à une vitesse de labour comprise entre 2 et $7 \mathrm{~km} / \mathrm{h}$. La profondeur de labour retenue a été différente selon l'état initial pour obtenir une même masse de terre humide travaillée dans les différentes situations expérimentales.

\section{Méthode de caractérisation des états structuraux}

La méthode de caractérisation des états utilisée avant et après labour est fondée sur l'observation morphologique et stratifiée du profil cultural (Manichon, 1982a) adaptée à une opération de fragmentation (Coulomb, 1991). On trouvera dans le tableau I les différentes caractéristiques des états internes des éléments structuraux distingués.

Pour disposer de variables quantifiées (Coulomb et al, 1993), nous avons procédé à l'interprétation stéréoscopique de photographies de la face du profil sur laquelle les différents types d'éléments structuraux et le pourtour des bandes avaient été identifiés sur le terrain. Les contours des différents objets sont reportés sur une feuille de papier à l'aide d'une pointe fine de taille définie $(0,7 \mathrm{~mm})$, numérisés grâce à une caméra vidéo $C C D$ associée à une carte $512 \times 512$ pixels. 
Tableau I. Caractéristiques des états internes distingués (Manichon, 1982a).

État interne Principales caractéristiques

\begin{tabular}{|c|c|c|}
\hline$\Delta$ & $\begin{array}{l}\text { Aspect continu } \\
\text { Faces de fragmentation peu r } \\
\text { Porosité visible très faible ou } \\
\text { Cohésion à sec élevée }\end{array}$ & uses de forme conchoïdale \\
\hline$\varnothing$ & \multicolumn{2}{|c|}{ État delta avec amorces de fissures sous l'action d'une fragmentation } \\
\hline$\Gamma$ & $\begin{array}{l}\text { Agrégats de morphologie vari } \\
\text { Rugosité importante après fra } \\
\text { Porosité structurale non nulle } \\
\text { Cohésion plus faible }\end{array}$ & $\begin{array}{l}\text { discernables dans les mottes } \\
\text { tation }\end{array}$ \\
\hline re fine & \multicolumn{2}{|c|}{ Agrégats individualisés de diamètre inférieur à $2 \mathrm{~cm}$} \\
\hline \multirow{2}{*}{\multicolumn{2}{|c|}{$\begin{array}{l}\text { Leur surface ainsi que leur plus grande longueur (ca- } \\
\text { libre) sont fournies par le logiciel d'analyse d'image. } \\
\text { Chaque catégorie morphologique (mottes de même } \\
\text { état interne ou de même taille) est caractérisée par la } \\
\text { somme des surfaces individuelles des éléments struc- } \\
\text { turaux qui la constituent. Cette surface est jugée repré- } \\
\text { sentative du volume scruté (Coulomb et al, 1993). Les } \\
\text { surfaces occupées par les mottes poreuses }(\Gamma \text { ) et la } \\
\text { terre fine n'ont pas été dissociées car un passage pro- } \\
\text { gressif de l'état de terre fine à l'état de mottes } \Gamma \text { sous } \\
\text { l'action du climat pendant la période d'observation était } \\
\text { admissible (Coulomb et al, 1993). }\end{array}$}} & $\begin{array}{l}\text { bour, sur la teneur surfacique des différents types } \\
\text { d'états internes et, enfin, sur la proportion de mottes } \\
\text { de gros calibres }(>20 \mathrm{~cm}) \text {. }\end{array}$ \\
\hline & & $\begin{array}{l}\text { Sur NT, I'horizon de reprise }(0-10 \mathrm{~cm}) \text { se distingue } \\
\text { peu de l'horizon labouré non repris }(10-30 \mathrm{~cm} \text { ). IIs } \\
\text { sont tous deux d'état fragmentaire. Globalement, NT } \\
\text { se caractérise par une teneur surfacique en mottes } \\
\text { poreuses }(\Gamma) \text { et terre fine supérieure à } 75 \% \text { et des } \\
\text { mottes compactées plus ou moins fissurées ( } \Delta \text { et } \varnothing \text { ) } \\
\text { de faible taille (tableau II). }\end{array}$ \\
\hline $\begin{array}{l}\text { action du } \\
\text { admissible }\end{array}$ & $\begin{array}{l}\text { t la période d'observation était } \\
\text { al, 1993). }\end{array}$ & $\begin{array}{l}\text { T1 et T2 sont constitués de deux horizons bien dif- } \\
\text { férenciés : une couche supérieures }(0-5 \mathrm{~cm}) \text { d'état } \\
\text { fragmentaire (petites mottes compactes) qui résulte } \\
\text { de l'opération de nivellement et une couche sous- } \\
\text { jacente d'environ } 20 \mathrm{~cm} \text { d'épaisseur d'état compact et } \\
\text { d'aspect continu créée par le compactage. Celui-ci } \\
\text { n'ayant pas été totalement homogène, il est possible } \\
\text { de décomposer l'état de cet horizon en zones mas- }\end{array}$ \\
\hline $\begin{array}{l}\text { iité ex } \\
\text { espor }\end{array}$ & $\begin{array}{l}\text { ée pour } \\
\text { lée par c }\end{array}$ & $\begin{array}{l}\text { sives considérées comme des grosses mottes. Globa- } \\
\text { lement, T1 et T2 se caractérisent par une forte teneur } \\
\text { surfacique en mottes } \Delta \text { et } \varnothing(>75 \%) \text { et des mottes de } \\
\text { gros calibres (tableau II). Ces dernières représentent } \\
\text { plus de } 90 \% \text { des mottes } \Delta \text { et } \varnothing \text {. }\end{array}$ \\
\hline
\end{tabular}

Tableau II. Caractérisation des états avant labour.

Teneur surfacique en mottes: moyenne (min-max)

\begin{tabular}{|c|c|c|c|c|c|}
\hline Traitement & $\begin{array}{c}\Gamma \\
\text { et terre fine }\end{array}$ & $\Delta$ & $\varnothing 1$ & $\varnothing 2$ & $>20 \mathrm{~cm}$ \\
\hline État initial & $\%$ & $\%$ & $\%$ & $\%$ & $\%(\Delta$ et $\varnothing)$ \\
\hline $\mathrm{NT}(n=19)$ & $79(59-91)$ & $5 \quad(0-17)$ & $6(0-13)$ & $7(1-18)$ & $11(0-78)$ \\
\hline $\mathrm{T} 1(n=4)$ & $23(20-26)$ & $33(17-48)$ & $38(21-52)$ & $2(1-3)$ & $91(89-92)$ \\
\hline $\mathrm{T} 2(n=4)$ & $18(10-25)$ & $63(8-85)$ & $16(0-64)$ & $0(0-0)$ & $91(88-94)$ \\
\hline
\end{tabular}


T1 se différencie de T2 par une fissuration plus développée (tableau II) et par une plus forte résistance pénétrométrique (environ une centaine de newtons en plus) de la couche $5-25 \mathrm{~cm}$ mesurée la veille du labour pour une humidité et une densité équivalentes (Coulomb et al, 1993).

\section{Compatibilité des mesures entre avant et après labour}

L'analyse de l'évolution de l'état structural du sol au cours du labour implique que la comparaison des états avant et après labour soit faite sur des volumes correspondant à la réelle masse de terre que l'on a travaillée. Pour contrôler ce point, le plus simple était de fixer la profondeur de travail à un niveau identifiable sur les profils de caractérisation de l'état initial tout en restant dans une gamme habituelle de profondeur de travail du sol. Nous avonc donc cherché à fixer la profondeur de labour au fond d'Ap, qui correspond au fond du labour le plus profond, car on sait le repérer par le changement de couleur de la terre. Cependant, en raison du caractère destructif des observations et des mesures, il était impossible d'adopter le même taux d'échantillonnage pour caractériser les situations avant et après le travail du sol. Cela pose les problèmes de la représentativité des profils initiaux et de la taille optimale de l'unité d'échantillonnage pour mettre en correspondance les états et quantifier les évolutions.

Des mesures systématiques de profondeur de changement de couleur effectuée avant labour, en 26 points de la parcelle, nous ont permis de conclure pour cette variable que les profils initiaux étaient représentatifs de la majorité de la surface de la parcelle excepté les 10 premiers mètres dans le sens du travail des outils. Dans cette zone, la profondeur d'Ap est plus forte (d'environ $6 \mathrm{~cm}$ ) par rapport à la moyenne évaluée sur les profils, nous en tiendrons compte dans la discussion des résultats.

À l'échelle du passage de charrue, qui constitue notre unité d'observation, nous avons constaté qu'il n'y avait pas stricte correspondance entre la profondeur de labour et celle du changement de couleur. Tous traitements confondus, le rapport entre ces deux profondeurs est compris entre 0,80 et 1,15; cela nous interdit d'analyser l'évolution à cette échelle. Par contre, à l'échelle du traitement (moyennes de 4 passages adjacents de charrue pour T1 et T2, 8 pour NT), le rapport est en moyenne de 1 sur T1 et NT, et 0,9 sur T2. À cette échelle, nous pouvons donc admettre pour chaque traitement la correspondance entre la profondeur du changement de couleur et la profondeur de labour. Dans T2, la profondeur de labour est légèrement inférieure à la profondeur du changement de couleur. Nous discuterons de la sensibilité des résultats à un tel écart.

En conclusion, malgré les réserves précédentes, nous admettons que les volumes caractérisés avant et après labour sont comparables et correspondent à la masse de terre travaillée.
Comme la variabilité des états structuraux intratraitement est inférieure à la variabilité entre les traitements avant labour (Coulomb et al, 1993), il est par ailleurs possible de comparer les évolutions entre les traitements à cette échelle. Dans la suite de l'analyse, une répétition représente 4 passages de charrue dans T1 et T2, 8 passages dans NT. La taille plus importante de la répétition dans NT se justifie par une variabilité intratraitement supérieure (Coulomb et al, 1993). Le nombre de répétitions est de 6 dans T1 et NT, de 3 dans T2.

\section{Quantification de l'évolution}

Dans la mesure où les systèmes observés avant et après labour sont comparables, les variations de la surface de la couche travaillée, de la surface occupée par différentes catégories morphologiques et $\mathrm{du}$ nombre de mottes sont indicatrices d'une évolution. L'évolution volumique d'une catégorie morphologique peut traduire une variation du volume global observé à la suite d'une variation de la porosité de cette catégorie, mais peut aussi correspondre à une évolution massique à la suite d'un transfert entre catégories. L'évolution du nombre de mottes d'une taille donnée est un indicateur de fragmentation car ce processus mécanique implique la création de nouvelles mottes de taille plus faible. II n'exclut pas la possibilité d'une absence d'évolution du nombre de mottes de certaines classes en raison de transferts entre classes.

Nous quantifions les rapports entre les variations de surface définies précédemment et la surface initiale de la couche travaillée. Notre analyse de l'évolution de l'état structural du sol repose sur une interprétation des différents bilans concernés.

La surface correspondant au volume théoriquement travaillé est calculée à partir des mesures de profondeur et de largeur de travail effectuées sur la muraille de chaque passage de charrue. On la compare à la surface occupée après travail par les bandes de labour.

Pour une catégorie donnée, $X$, le bilan surfacique est exprimé par le rapport $B_{X}$ suivant :

$$
B X=(S 2 X-S 1 X) / S t
$$

$S 1 X$ : surface $X$ avant labour; $S 2 X$ : surface $X$ après labour; St: surface de la couche travaillée avant labour.

Nous calculons de même la variation du nombre total de mottes tout en sachant que, dans ce cas, la représentativité de notre observation est probablement plus faible qu'en terme de surface occupée (Coster et Cherman, 1989), et notre analyse risque d'être moins pertinente. Nous comparons par ailleurs les distributions en nombre des calibres des mottes avant et après labour. Nous avons testé leur similitude par un test du $\chi^{2}$ effectué sur les effectifs réellement observés sur le terrain. 


\section{RÉSULTATS}

\section{Évolution du volume de l'horizon labouré}

\section{Évolution du volume apparent}

La variation de surface de la couche labourée est positive dans tous les traitements. L'augmentation semble plus importante dans les traitements tassés ( $T 1$ et $\mathrm{T} 2$ ) que dans le traitement non tassé NT. C'est en particulier le cas pour un profil de T1 (fig 1). Cette variation de surface peut être le reflet d'une variation de l'importance de plusieurs compartiments :

- les mottes d'état interne $\Delta$ ou $ø$;

- les mottes $\Gamma$ et la terre fine;

- les vides de plus de $1 \mathrm{~cm}$ internes à la bande labour.

\section{Décomposition des variations de volume apparent selon les différents éléments structuraux du sol}

Les variations des surfaces occupées par les mottes d'état interne $\Delta$ et $\varnothing$ par rapport à la surface initiale de la couche travaillée sont faibles dans tous les profils (tableau III). Elles sont en moyenne inférieures à $3 \%$ en valeur absolue dans $\mathrm{T} 1$ et $\mathrm{NT}$, et toujours inférieures à $6 \%$ sauf pour le profil de T1 distingué précédemment pour sa forte variation de volume apparent (fig 1). Dans ce profil l'augmentation de surface des mottes $\Delta$ et $\emptyset$ est apparemment de $13 \%$. Dans T2, la surface en $\Delta$ et $\varnothing$ semble diminuer en moyenne de $7 \%$. Cette diminution est toujours inférieure à $12 \%$.

En revanche, on constate une augmentation nette de la surface occupée par les mottes $\Gamma$ et la

Tableau III. Bilans des surfaces occupées par les différentes fractions.

Variation relative de surface (\%) moyenne (min-max)

\begin{tabular}{cccc} 
Traitement & $\begin{array}{c}\text { Mottes } \\
\Delta \text { et } \varnothing\end{array}$ & $\begin{array}{c}\text { Mottes } \\
\Gamma \text { et terre fine }\end{array}$ & $\begin{array}{c}\text { Vides internes } \\
\geq 1 \mathrm{~cm}\end{array}$ \\
& & & \\
NT $(n=6)$ & $-1(-6 ;+2)$ & $+12(+8 ;+18)$ & $0(0 ; 0)$ \\
T1 $(n=6)$ & $+3(-4 ;+13)$ & $+19(+15 ;+23)$ & $+3(+1 ;+4)$ \\
T2 $(n=3)$ & $-7(-12 ;-5)$ & $+35(+33 ;+36)$ & $+4(+2 ;+6)$ \\
\hline
\end{tabular}

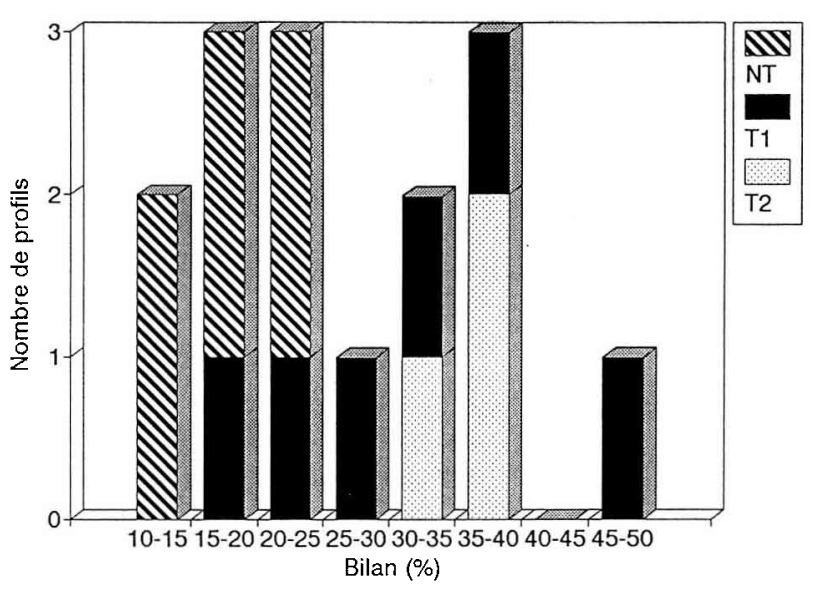

Fig 1. Histogramme de la variation (en \%) de surface de la bande labourée au cours du labour rapportée à la surface initiale de la bande avant labour (bilan). $N T$; $\square T 1$;

terre fine, soit 8 à $18 \%$ dans NT, 15 à $23 \%$ dans $\mathrm{T} 1$, plus de $30 \%$ dans T2. Dans $\mathrm{T} 1$ et T2, il apparaît de surcroît des vides internes à la bande de labour, qui occupent jusqu'à $6 \%$ de la surface.

\section{Discussion}

Les bilans effectués semblent indiquer que la part des mottes d'états internes $\Delta$ et $\varnothing$ évolue globalement peu au cours du labour alors que celle des mottes $\Gamma$ et de la terre fine augmente, quel que soit l'état structural intial. L'augmentation est supérieure lorsque ce dernier est continu et tassé. Dans ce cas, elle s'accompagne d'un accroissement de l'espace entre les mottes, lié à l'apparition de vides supracentimétriques.

L'hypothèse d'une variation nulle du volume correspondant aux mottes d'état interne $\Delta$ et $\varnothing$ n'est toutefois acceptable que si l'erreur sur le calcul de la variation relative de surface est supérieure aux variations observées.

Pour une erreur relative d'évaluation des surfaces de $\pm 5 \%$ l'erreur sur le bilan surfacique est en moyenne de $\pm 8 \%$ pour les états initialement tassés, de $\pm 2 \%$ pour NT.

Notre hypothèse n'est donc pas remise en cause dans NT et T1, sauf pour un profil de NT (légère diminution de surface de mottes $\Delta$ et $\varnothing$ de $6 \%$ ) et pour le profil de T1 situé dans la zone où le changement de couleur est un peu plus profond que dans le reste de la parcelle (augmentation de surface des mottes $\Delta$ et $\varnothing$ de $13 \%$ ). Dans ce dernier cas, le tassement d'Ap a 
probablement entraîné la création d'un volume initial en mottes $\Delta$ supérieur à celui que nous avons estimé, volume retrouvé après labour si l'on admet que le soc de la charrue a suivi la cote du changement de couleur. Dans T2, le bilan ne permet pas d'exclure une légère diminution de la surface en $\Delta$ et $\varnothing$. Mais comme le labour y est moins profond que la cote du changement de couleur, ce résultat pourrait n'être qu'un artefact en raison d'une surestimation de la surface en $\Delta$ et $\varnothing$ avant labour. Nous avons donc réévalué le bilan en calculant la surface en $\Delta$ et $\varnothing$ avant labour sur la profondeur effective de travail, et non pas sur celle du changement de couleur. Le bilan devient inférieur à $4 \%$ en valeur absolue pour tous les profils. II est en moyenne de $1 \%$. Nous pouvons donc aussi admettre l'absence de variation de volume des mottes d'état interne $\Delta$ et $\varnothing$ dans $T 2$.

L'erreur sur la variation de surface des mottes $\Gamma$ et de la terre fine est inférieure à $10 \%$ pour tous les états structuraux initiaux, aussi nous pouvons accepter l'hypothèse d'une augmentation du volume de cette catégorie.

D'après ces résultats, l'augmentation du volume total de l'horizon labouré ne peut avoir pour origine une variation de la porosité des mottes $\Delta$ et $\oslash$. Dans NT, elle ne peut être liée qu'à l'augmentation de la porosité du compartiment constitué par les mottes $\Gamma$ et la terre fine, puisqu'aucun espace poral $\geq 1 \mathrm{~cm}$ n'apparaît. Dans $T 1$ et $T 2$, le volume des mottes $\Gamma$ et de la terre fine augmente mais ce phénomène s'accompagne de l'apparition d'un espace poral supracentimétrique qui ne peut être expliquée que par une variation de l'arrangement relatif des mottes $\Delta$ et $\varnothing$ les unes par rapport aux autres. L'état structural initial conditionne donc la taille de l'espace poral créé au cours du labour.

Tableau IV. Bilans des surfaces occupées par les mottes delta selon leur niveau de fissuration.

Variation relative de surface des mottes (\%) moyenne (min-max)

$\begin{array}{lccc}\text { Traitement } & \varnothing 2 & \varnothing 1 & \Delta \\ \text { NT }(n=6) & -1(-5 ;+5) & -5(-3 ;-5) & +4(+1 ;+6) \\ \text { T1 }(n=6) & +17(+4 ;+31) & -17(-4 ;-29) & +3(-13 ;+15) \\ \text { T2 }(n=3) & +6(+4 ;+10) & +3(-6 ;+9) & -16(-8 ;-25)\end{array}$

\section{Évolution de l'état de fissuration des mottes}

\section{Bilan}

L'absence de variation significative $\mathrm{du}$ volume occupé globalement par les mottes $\Delta$ et $\varnothing$ n'exclut pas la possibilité d'une évolution de leur porosité de fissures.

L'état de fissuration des mottes varie effectivement au cours du labour (tableau IV). Dans T1 et T2, des mottes $\varnothing$ à 2 niveaux (tableau I) apparaissent au cours du labour. L'augmentation de surface correspondante est plus importante dans $\mathrm{T} 1$ que dans T2. Elle est associée à une diminution de la surface occupée par les mottes ø à 1 niveau dans T1, des mottes $\Delta$ dans T2. Dans NT, la surface occupée par les mottes $ø$ diminue alors que celle des mottes $\Delta$ augmente.

Bien que la surface du compartiment structural composé des mottes $\Delta$ et $\varnothing$ ne varie pas, ce compartiment n'est donc pas indemne d'évolutions, puisque les parts respectives des mottes fissurées à des niveaux différents évoluent au sein du compartiment.

\section{Discussion}

L'état initial paraît donc conditionner l'évolution des états de fissuration des mottes. Les processus mécaniques en cause sont probablement de natures différentes suivant l'état initial. Dans T1 et T2, l'augmentation de la fissuration pourrait être indicatrice d'un travail de fragmentation, alors que dans NT la diminution de la fissuration pourrait être indicatrice d'un compactage.

Ce compactage pourrait résulter :

- d'un tassement, par la roue de raie, de la base de la bande de labour correspondant au passage de charrue précédent ;

- d'un compactage propre au travail de la charrue ;

- d'un tassement par la roue de guéret du passage précédent.

La dernière hypothèse implique l'augmentation de la teneur en $\Delta$ pour les bandes correspondant au passage de la roue de guéret. Cela n'a pas été constaté visuellement. Elle est donc peu probable. Par contre, si l'on recalcule le bilan sans tenir compte de la surface occupée par les mottes $\Delta$ situées sous la roue de raie après labour, il devient égal à $0,5 \%$ en moyenne et demeure inférieur à $2 \%$ dans tous les cas. La varia- 
tion devient donc pratiquement nulle et d'un ordre de grandeur équivalent à celui de l'erreur calculée sur le bilan. L'origine du $\Delta$ néoformé dans NT peut raisonnablement être attribuée à une modification de l'état final par compactage sous la roue de raie.

Dans $T 1$ et $T 2$, ce phénomène n'apparaît pas alors que le dégagement de la raie y est plus mauvais. Trois raisons pourraient expliquer ce résultat :

- les mottes situées sous la roue de raie étaient déjà d'état interne $\Delta$ avant le passage de celleci ;

- le débordement de la terre par-dessus le versoir est moins important lorsque l'état est initialement tassé : cela diminue la probabilité de tasser de la terre fine dont la proportion était déjà moins forte dans l'état initial ;

- la taille des éléments concernés par ce débordement est trop importante pour que leur état interne soit affecté par le passage de la roue de raie ; en effet, on a déterminé au laboratoire de science du sol INRA d'Avignon l'existence d'un calibre limite pour la création de l'état $\Delta$ par compactage lors de compressions uniaxiales et confinées, drainées ou non, à chargements uniques ou cycliques (De Leon, 1991).

\section{Évolution des calibres des éléments structuraux}

\section{Évolution du nombre de mottes d'un calibre donné}

Le nombre total de mottes est multiplié par 2,8 au cours du labour dans T1 et T2, alors qu'il varie peu dans NT (tableau V).

Dans $T 1$ et $T 2$, les distributions des calibres avant et après labour sont significativement diffé-

Tableau V. Évolution du nombre total de mottes au cours du labour.

\begin{tabular}{ccc} 
Traitement & \multicolumn{2}{c}{$\begin{array}{c}\text { Nombre total de mottes } \\
\text { à l'échelle de } 4 \text { passages }\end{array}$} \\
\cline { 2 - 3 } & Avant labour & Après labour \\
\hline NT & 83 & \\
T1 & 68 & 76 \\
T2 & 62 & 192 \\
\hline
\end{tabular}

rentes au niveau $1 \%$ (test du $\chi^{2}$ ). L'augmentation du nombre de mottes dans T1 et T2 est donc associée à une modification de la distribution des calibres (figs 2 et 3 ). Les effectifs de toutes les classes de calibre inférieur à $35 \mathrm{~cm}$ augmentent. On constate, en particulier dans $\mathrm{T} 2$, une apparition de mottes entre 10 et $25 \mathrm{~cm}$. Dans T1, l'augmentation du nombre de mottes de moins de $5 \mathrm{~cm}$ est plus forte; mais l'évolution de l'étendue de la distribution des calibres est beaucoup plus faible qu'en T2 (figs 2 et 3 ). Dans NT, la distribution des mottes ne varie pas significativement si l'on ne tient pas compte de la répartition des mottes de plus de $20 \mathrm{~cm}$ dont l'apparition est liée au compactage par la roue de raie.

\section{Discussion}

Les résultats montrent que l'évolution du nombre de mottes est dépendante de l'état structural initial. On peut donc penser que les mécanismes de fragmentation en jeu au cours du labour diffèrent selon l'état initial.

Dans NT, le calibre des mottes inférieures à la largeur de prise de raie ne varie pas ou peu au cours du labour. La fragmentation semble donc se limiter à une érosion légère du pourtour des mottes. Sous cette hypothèse, le nombre total de mottes varie peu, et seules les classes de faible calibre (qui disparaissent) ou de fort calibre (supérieur à la largeur de la bande de labour) sont concernées, ce qui est concordant avec nos résultats.

Dans T1 et T2, l'augmentation très importante du nombre de mottes dans les classes 5 à $25 \mathrm{~cm}$ ne peut s'expliquer par un tel mécanisme. Une division des mottes en mottes de plus faible taille d'état interne $\Delta$, accompagnée ou non d'une érosion légère du pourtour des mottes résultant du frottement des mottes le long du versoir et entre elles, serait en revanche un mécanisme vraisemblable. II se produirait dans ce cas un réel travail de fragmentation des mottes. $\mathrm{Si}$ l'énergie fournie est insuffisante pour assurer une division complète de toutes les mottes lors du labour, le travail se traduirait par une augmentation du nombre de mottes fissurées. Les mottes concernées seraient plutôt de fort calibre.

On constate que le nombre de mottes $\Delta$ a plus que triplé au cours du labour alors que celui des mottes fissurées augmente plus faiblement 


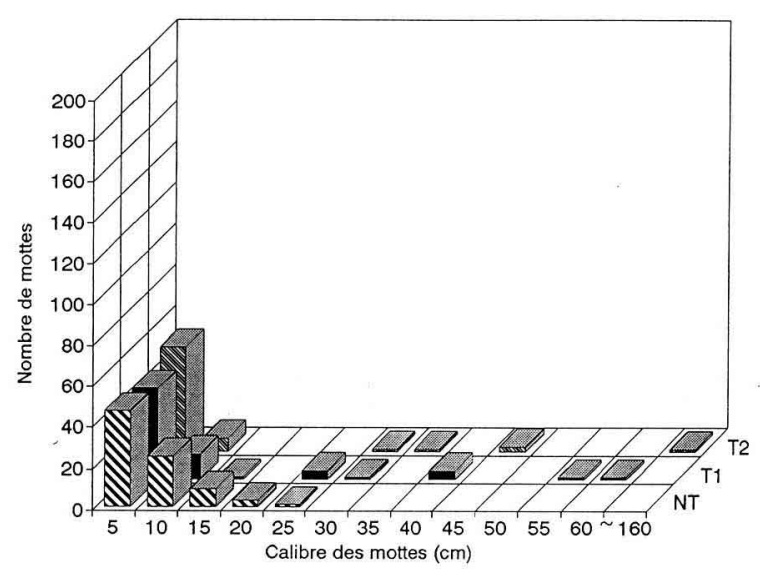

Fig 2. Distribution des calibres des mottes repérées sur 4 passages de charrue avant labour.

Tableau VI. Évolution du nombre de mottes selon leur niveau de fissuration sur T1 et T2.

\begin{tabular}{lrrr}
$\begin{array}{l}\text { Nombre total de mottes } \\
\text { à l'échelle de 4 passages }\end{array}$ & $\Delta$ & $\varnothing 1$ & $\varnothing 2$ \\
\hline Avant labour & 47 & 15 & 4 \\
Après labour & 162 & 17 & 8 \\
\hline
\end{tabular}

(tableau VI). Les fissures de 2 niveaux apparaissent sur les mottes de plus de $10 \mathrm{~cm}$. Nos hypothèses sont donc plausibles.

\section{DISCUSSION GÉNÉRALE ET CONCLUSION}

L'analyse quantitative par bilan que nous venons d'effectuer montre que les processus de fragmentation au cours du labour dépendent de l'état structural initial du sol. Elle rend plausible les hypothèses formulées sur ces processus.

Le labour se traduit non seulement par une augmentation de volume de la couche de sol, mais aussi par une modification de l'organisation spatiale des phases solides et porales, qui dépend de l'état initial du sol.

Selon cet état initial, nous avons précisé les éléments structuraux affectés et nous avons quantifié l'importance des phénomènes dans les conditions expérimentales explorées.

Quel que soit l'état structural du sol, la porosité du compartiment constitué par les mottes $\Gamma$ et la terre fine augmente au cours du labour modifiant le volume apparent de la couche labourée alors que, globablement, le volume des mottes $\Delta$ et $\varnothing$ ne varie pas significativement.

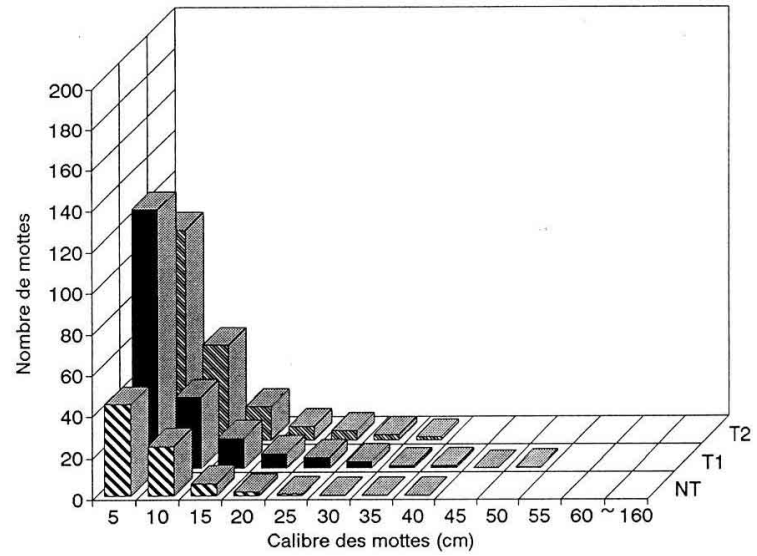

Fig 3. Distribution des calibres des mottes repérées sur 4 passages de charrue après labour.

Cette augmentation de l'espace poral dans la couche labourée est plus importante lorsque l'état est initialement continu et tassé. Cela s'explique en partie par l'apparition d'un espace poral supracentimétrique supplémentaire à la suite d'une réorganisation de la phase solide. La réduction du calibre des mottes modifie en effet le champ des possibilités de réorganisation. Un tel phénomène est impossible lorsque l'état initial est fragmentaire, puisque le calibre des mottes varie peu.

L'état interne des mottes est modifié sans que cela se traduise par une évolution du volume global :

- une fissuration se développe sur les grosses mottes $\Delta$ lorsque l'état est initialement continu et tassé ;

- une création de mottes $\Delta$ par compactage sous la roue de raie peut intervenir en état fragmentaire.

Le bilan effectué sur la surface occupée par les mottes $\Delta$ et $\varnothing$ ne rend pas compte de la création de mottes $\Delta$ par compactage. Les raisons que l'on peut invoquer sont la disparition simultanée de mottes $\varnothing$ de faible calibre par érosion, à la suite d'un transfert dans le compartiment terre fine, et les faibles ampleurs des phénomènes concernés, qui demeurent de l'ordre de l'erreur que l'on peut faire sur le bilan.

Des éléments de modélisation du comportement du sol au labour pour la texture et l'humidité du sol explorées ressortent de notre analyse.

Lorsque l'état initial est fragmentaire, le labour a une fonction de découpe, déplacement et augmentation de la porosité entre des éléments déjà individualisés, dont le calibre est peu modifié et 
l'état interne ne change pas, sauf création de mottes $\Delta$ par compactage. Son action sur la modification de l'état structural ne concerne donc que l'arrangement entre les mottes.

Lorsque l'état initial est tassé et d'aspect continu, le labour a aussi pour fonction de modifier la taille et l'état de fissuration des mottes à la suite probablement d'un processus de division inexistant en état fragmentaire. L'ampleur de cette réduction de calibre n'est pas la même pour deux états compactés différenciés par l'état de fissuration des mottes et la résistance mécanique de la couche labourée. Elle est plus faible pour l'état où les mottes sont initialement les plus fissurées, alors même que la présence de fissures, zones de ruptures préférentielles, devrait accentuer la réduction de calibre des mottes. Mais la résistance pénétrométrique de l'état le plus fissuré est plus forte sur la couche labourée ce qui explique probablement le résultat observé.

L'application de notre méthode d'évaluation à une gamme plus large d'états non fragmentaires devrait permettre dans l'avenir de fournir un modèle d'évolution des états structuraux au labour dans la mesure où elle s'est avérée pertinente pour dissocier les évolutions dans le cas des états initiaux contrastés que nous avons étudiés.

\section{RÉFÉRENCES}

Coster M, Chermant J (1989) Précis d'analyse d'images. CNRS plus, CNRS, pp 545

Coulomb I (1991) Analyse quantitative du comportement du sol au labour : rôle de l'état structural initial. Thèse INA-PG, Paris, pp $230+$ annexes

Coulomb I, Caneill J, Manichon H (1993) Comportement du sol au labour : méthode d'analyse et évaluation des conséquences de l'état initial du sol sur l'état transformé par le labour. agronomie 13, 45-46

De Leon F (1991) Morphologie - Propriétés physiques et conditions de formation des éléments structuraux de la couche labourée. Contribution à l'interprétation du profil cultural. Thèse INA-PG, pp 133 + annexes

Manichon H (1982a) Influence des systèmes de culture sur le profil cultural : élaboration d'une méthode de diagnostic basée sur l'observation morphologique. Thèse docteur ingénieur INA-PG, Paris, pp 214 + annexes

Manichon H (1988) Compactage, décompactage du sol et systèmes de culture. CR Acad Agric Fr 74 , 43-54 